\title{
Further Studies in the Annealing of a Borosilicate Glass
}

\author{
Sam Spinner and Albert Napolitano \\ Institute for Materials Research, National Bureau of Standards, Washington, D.C.
}

(December 22, 1965)

\begin{abstract}
Time-index of refraction isotherms for a borosilicate glass in the transformation region have been established. By comparing two quenched glasses of the same index, one quenched after attaining equilibrium at a given temperature and the other quenched from a temperature at which the specimen was approaching a higher value of index, curves similar to the one obtained by Ritland in comparing rate cooled and quenched specimens are developed.
\end{abstract}

Key Words: Annealing, borosilicate glass, glass, time-index of refraction isotherms, transformation range, index of refraction.

\section{Introduction}

Ritland $[1]^{1}$ has demonstrated that glasses of the same composition and refractive index can nevertheless be quite different in other respects, such as expansivity, electrical resistivity, etc, depending upon whether the index was achieved by cooling the glass at some (relatively slow) constant rate through the transformation range ("rate cooled"), or by cooling the glass rapidly from some holding temperature within the transformation range, this temperature being maintained for a time sufficient for the glass to stabilize at that temperature ("soaked"). This demonstrated the inadequacy of certain aspects of the fictive temperature concept which assumes that the structural state of a glass can be completely described by a single ordering parameter. Ritland also pointed out that Tool [14], who originated the fictive temperature concept, was not guilty of this over-simplification, recognizing that although every temperature in the transformation range corresponds to a certain equilibrium ${ }^{2}$ state, "it does not necessarily follow that every nonequilibrium state is identical with one of these."

One of the several ways by which Ritland demonstrated the difference between rate cooled and soaked specimens (and the one which forms the starting point of the present investigation) is shown in figure 1 , which is taken from his paper (fig. 3, of ref. 1). When a rate cooled specimen is placed in a furnace held at the tem-

${ }^{1}$ Figures in brackets indicate literature references at end of this paper.

2The word "equilibrium" as used in this paper is, of course, not to be interpreted in the fullest thermodynamic sense, but rather, in the more limited sense of the condition of a glass at which the structure in the transformation range has stabilized, so that its properties no longer change within reasonable time [2]. perature required to maintain the same index as for a soaked specimen, the index of the rate cooled specimen is seen to dip rather quickly before returning more slowly to its initial value. Clearly, had the rate cooled specimen been initially in the same structural state as the soaked specimen of the same index, then this dip in index before returning to its initial value would not have taken place; instead, the rate cooled specimen would follow the same flat line as the soaked specimen.

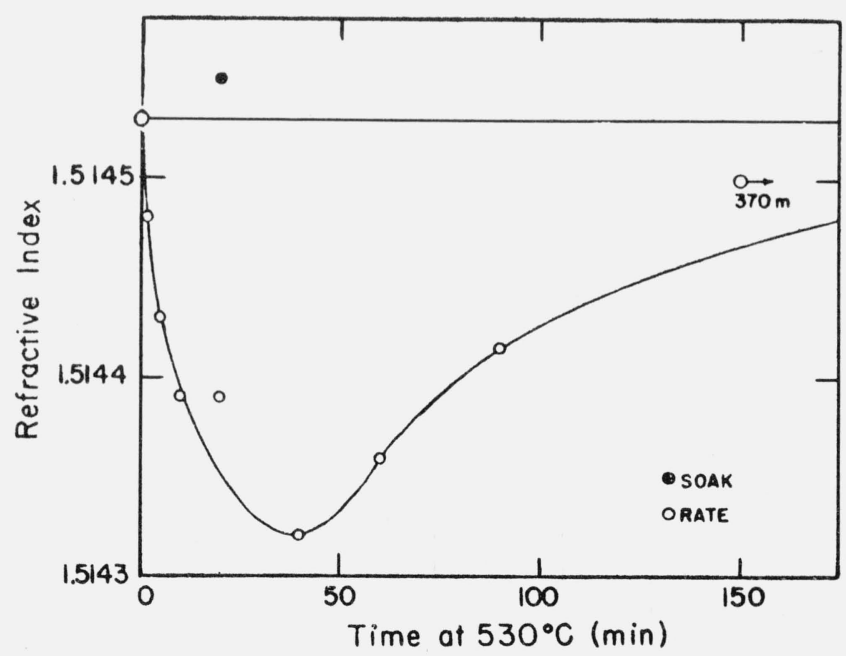

FIGURE 1. Room temperature refractive index as a function of treatment time at $530^{\circ} \mathrm{C}$. Figure 3 from Ritland [1]. 
In an attempt to shed further light on this problem of the relation between the condition of a glass and its thermal history, the present investigation takes the following line of approach. It is known that the lower the temperature at which a glass is held in the annealing region, the higher the room-temperature equilibrium value of refractive index that will be reached. Then, the same room temperature value of index for two glasses can be obtained not only by soaking and appropriate rate cooling, but also using only suddenly cooled (quenched) glasses; one glass being quenched from an equilibrium index at some given temperature (corresponding to Ritland's soak specimen) and the other glass being quenched from some lower temperature, but at that instant in its approach to its equilibrium, when its index equaled that of the specimen already at equilibrium. We, then, propose to compare two such quenched specimens in a manner similar to that used by Ritland, as described above in comparing soaked and rate cooled specimens. Such an experimental approach should be revealing since, figuratively speaking, one freezes the structure of the glass in its approach to equilibrium; and the nature of this approach to equilibrium is recognized to be of fundamental importance in understanding the processes taking place in the glass transformation range.

Kovacs [3] has already performed an experiment which, though quite different in detail, is similar in principle to the one proposed here. His test material was an organic polymer having a transformation range from about 10 to $40{ }^{\circ} \mathrm{C}$ and the property measured was the change in volume. It will be shown that the results obtained in this investigation, using an oxide glass, having a transformation range from about 490 to $605^{\circ} \mathrm{C}$, with refractive index as the indicating property, are in qualitative agreement with those of Kovacs.

\section{Experimental Procedure}

\subsection{Specimens}

The glass used in this study was a borosilicate crown, BSC 517. This is the same type of glass used in a previous study on annealing and is also very similar in composition to the one used by Ritland. All the specimens were cut from the same large block, to about $1 \mathrm{~cm} \times 1 \mathrm{~cm} \times 0.1 \mathrm{~cm}$.

There are several advantages in the use of such small specimens. First, no appreciable strain is introduced into the specimens when they are subjected to the sudden temperature changes which the experimental procedure requires, so that this possibly disturbing factor does not affect the measurement of index nor the pattern of the data to be shown. Second, and related to this, the danger of breakage of specimens in the course of the experiment is lessened. Actually, some of the specimens did break after repeated heating and cooling, but, if necessary, these were replaced by other specimens without loss in the continuity of the data. Also, as long as one square corner remained, it was still possible to measure the index of a broken specimen on the refractometer (described in the next section). Third, the times required for a specimen to come to thermal equilibrium, when suddenly introduced into, or removed from the furnace are quite short. Using relations developed by Brandt [4], the time taken for the central layer of a specimen of the size used here to come to within one degree of the surface temperature when subjected to such sudden temperature changes (of about $500{ }^{\circ} \mathrm{C}$ ) is computed to be less than 5 sec. These relations assume that the ambient temperature does not change in the vicinity of the specimen. Since this condition was not fully realized for the quenched specimens, the time for the specimens to reach room temperature is somewhat longer than this. However, this is unimportant since the time for cooling of the specimens through the transformation region is well within this figure.

\subsection{Apparatus}

\section{a. Refractometer}

All measurements of index, using the sodium D line, were made on the Grauer refractometer previously described [5]. It will only be recalled here that if reasonable care is taken in cutting the corners of the specimens square (no grinding or polishing of the specimens is necessary), then absolute values of index can be measured to \pm 2 in the fifth decimal place; and differencies in values of index, which this study mainly utilized, can be determined to better than \pm 1 in the fifth decimal place.

\section{b. Furnace}

A sketch of the furnace used is shown in figure 2 . Its main feature is a cylindrical metal slab, shown in the upper right-hand corner of the figure, containing ten radially placed slots which serve as receptacles for the specimens. Phosphor bronze metal is used to prevent scaling and serves to reduce thermal gradients between specimens in the slots. The slab can be rotated in the furnace by means of a ceramic rod attached to its center and extending vertically outside the furnace. When a specimen in a slot in the rotary slab lines up with the exit slot, appropriately cut in a stationary metal plate on which the rotary slab rests, the specimen drops from the furnace. [The expression "drops from the furnace" thus refers to the sudden drop in temperature which the specimen undergoes when it is air-quenched as well as the physical dropping from the furnace.] A detent mechanism permits any of the ten slots in the rotary slab to line up exactly with the exit slot. The specimens can be introduced into any of the slots in the rotary slab in a similar manner by being dropped from above the furnace, through a long entry slot cut in a ceramic cylinder which rests on the rotary slab. This cylinder can also be rotated so that the entry slot lines up with any of the slots in the rotary slab. The advantage in time saving and flexibility of being able to heat nine specimens simultaneously, if desired, is obvious. 


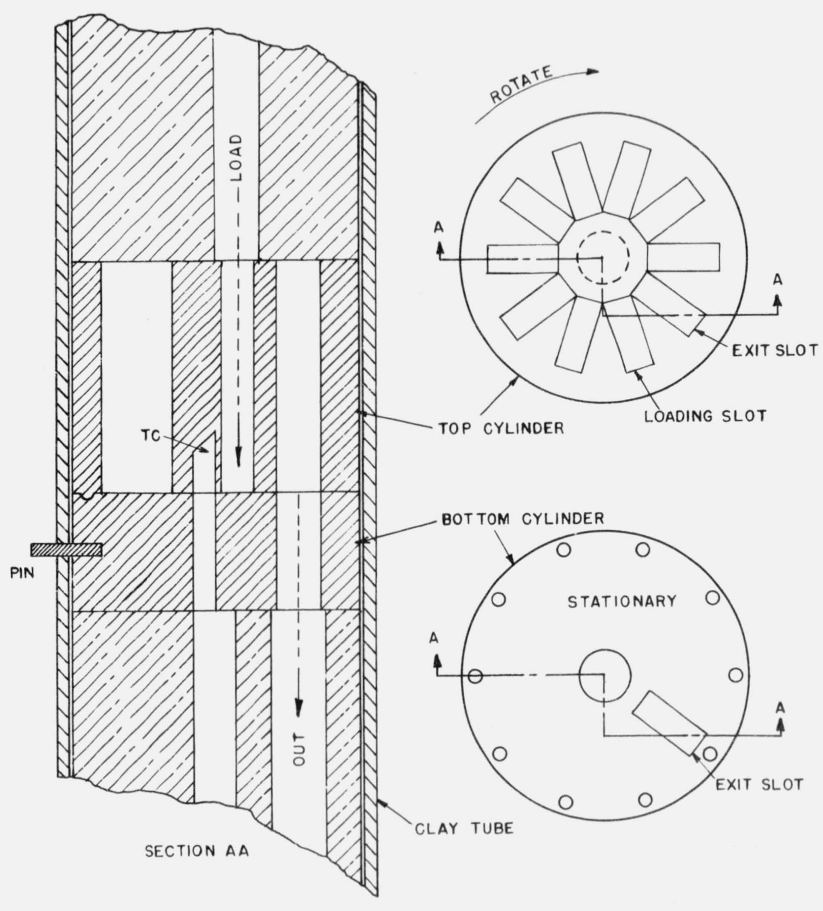

Figure 2. Cross-sectional sketch of rotary furnace.

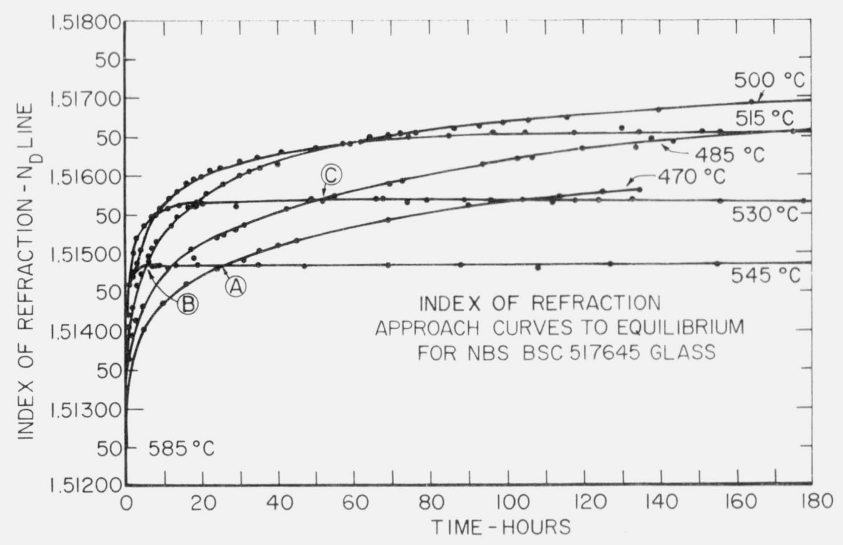

Figure 3. Time, index isotherms for BSC 517. Indices measured at room temperature.

intervals in the furnace between index measurements; yet the curves are quite smooth. Had the time for establishing thermal equilibrium been significant, then the scatter between points based on these different time intervals in the furnace between index measurements would have been greater than actually was the case.

\section{Results}

The time-index isotherms for various temperatures in the transformation range of the glass used here are shown in figure 3. Similar curves have been obtained by other investigators [7, 8, 9, 10]. Roughly similar curves can also be established in which the equilibrium values are reached from above rather than below. Daragon, [10] for instance, has obtained curves in which equilibrium is approached from higher as well as lower values, using density as the indicating parameter. It is experimentally more convenient, mainly because of the shorter times involved, to go from lower to higher values of index, as was done here.

Figure 4 shows the equilibrium index as a function of the equilibrium temperature in the transformation range, based on the data in figure 3 . The linear relationship is the usual one for multicomponent oxide glasses, and enables one to readily select the annealing temperature required to obtain a given index.

The general characteristic of the curves in figure 3 is seen to be that the higher the temperatures used the more rapid the rise to a lower equilibrium index. At the lowest temperatures (below $500{ }^{\circ} \mathrm{C}$ ) equilibrium was not achieved, the index continuing to rise slightly but significantly after seven weeks. We now designate the flat portion of each curve as the "equilibrium line" and the preceding portion of each line as the "approach curve." It is seen from the figure that the pattern of these curves leads to a series of crossover points at which an approach curve to a higher 


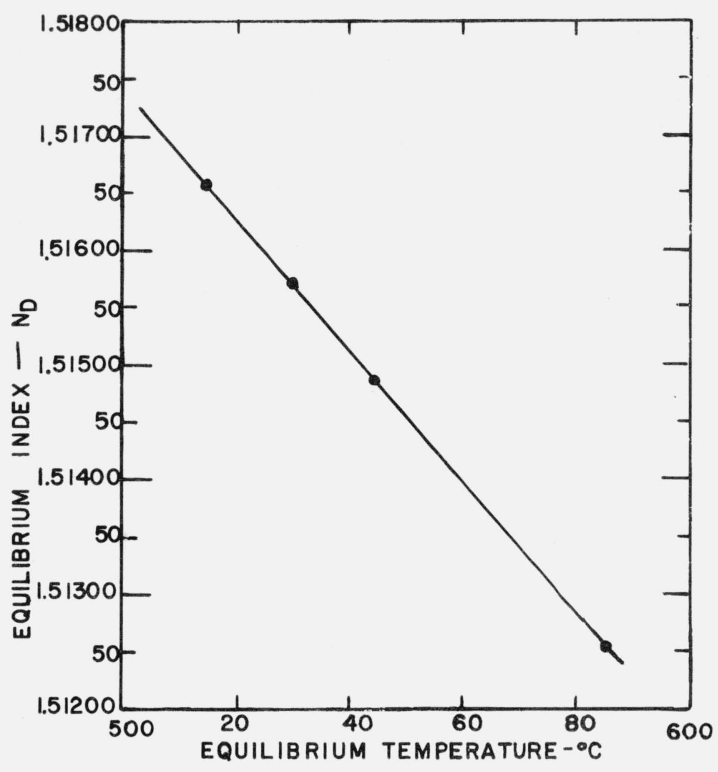

Figure 4. Equilibrium index as a function of temperature for BSC 517

equilibrium index (at a lower temperature) intersects either an equilibrium line at a lower index (higher temperature) or another faster approach curve, also at a higher temperature. Following now, the approach already indicated, we select certain of these crossover points, and interrupt the approach curve at the moment when the index of the specimen reaches the value of the equilibrium line. The temperature of the furnace is then reset as closely as practicable to the original value for the equilibrium line and the specimen is reinserted at this new temperature. Successive index readings are then taken after short intervals in the furnace so that slight effects will not be overlooked. The crossovers selected for this are shown as A, B, and $\mathrm{C}$ in figure 3 . Figure 5 shows the index-time changes following this procedure for crossover points $A$ and $B$, in which two different approach curves $\left(470\right.$ and $\left.500{ }^{\circ} \mathrm{C}\right)$ cross the same equilibrium line $\left(545{ }^{\circ} \mathrm{C}\right)$ and figure 6 shows the results of the $477{ }^{\circ} \mathrm{C}$ approach curve meeting the 527 ${ }^{\circ} \mathrm{C}$ equilibrium line.

In figure 5 , in order to check the reproducibility of the data, three specimens from the approach curve were selected, one at an index slightly below, one above, and one as close as possible to the index at equilibrium. The three resulting curves are seen to be similar to each other. The curves are seen to approach the equilibrium line at a slightly higher value than the original one, due to the fact that the resetting of the temperature controller resulted in a slightly lower temperature and, hence, a slightly higher value of index. This is demonstrated by the control specimen shown at the left retained at the original equilibrium index, also coming to the new value of index. The lines are drawn (mainly) through the experimental points primarily to distinguish these three sets of points.

Once the similarity of the three sets of points was demonstrated in figure 5 , only one set was used to show the data in figure 6 . In this figure also, the temperatures (approach temperature, $477^{\circ} \mathrm{C}$; equilibrium temperature, $527^{\circ} \mathrm{C}$ ) are seen to be somewhat different

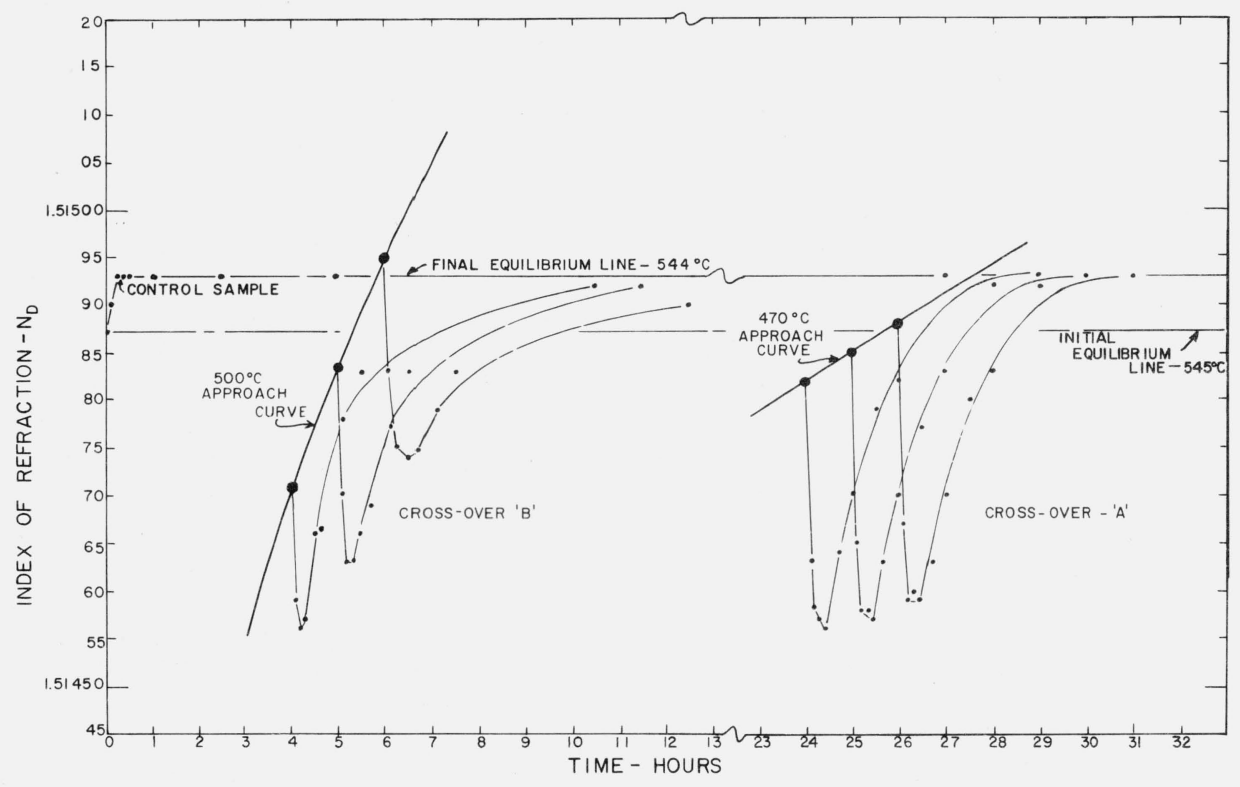

Figure 5. Index versus time at crossovers $A$ and $B$.

Equilibrium temperature for both crossovers is $545{ }^{\circ} \mathrm{C}$. Approach temperature for crossover A is $470{ }^{\circ} \mathrm{C}$, for crossover B, $500^{\circ} \mathrm{C}$. 
from the corresponding ones in figure 3 , as the original temperatures were not duplicated exactly to establish the data for this crossover. The similarity of the data in figures 5 and 6 for the specimens from the approach curve to Ritland's results for the rate cooled specimens is quite clear.

An additional experiment was designed to compare a specimen held for a relatively long time at equilibrium, with one held for a considerably shorter time at equilibrium. The test for this comparison was the characteristic of the curve of the descent to a lower equilibrium index. A third specimen at the same index, but on an approach curve to a higher index (an approach specimen) was included in the comparison. The two curves from the equilibrium line agreed within experimental error, indicating no difference in specimens held for longer or shorter times at equilibrium; however, the approach specimen descended more rapidly than the other two to the new value of index. The similarity in the characteristics of the two equilibrium specimens indicates that in comparing an approach specimen with an equilibrium specimen of the same index, it is not necessary to choose an equilibrium specimen at that instant when it is intersected by the approach specimen. An equilibrium specimen may be selected from any point along its time base. Furthermore, in those cases where no intersection is observed at all (this occurs more often

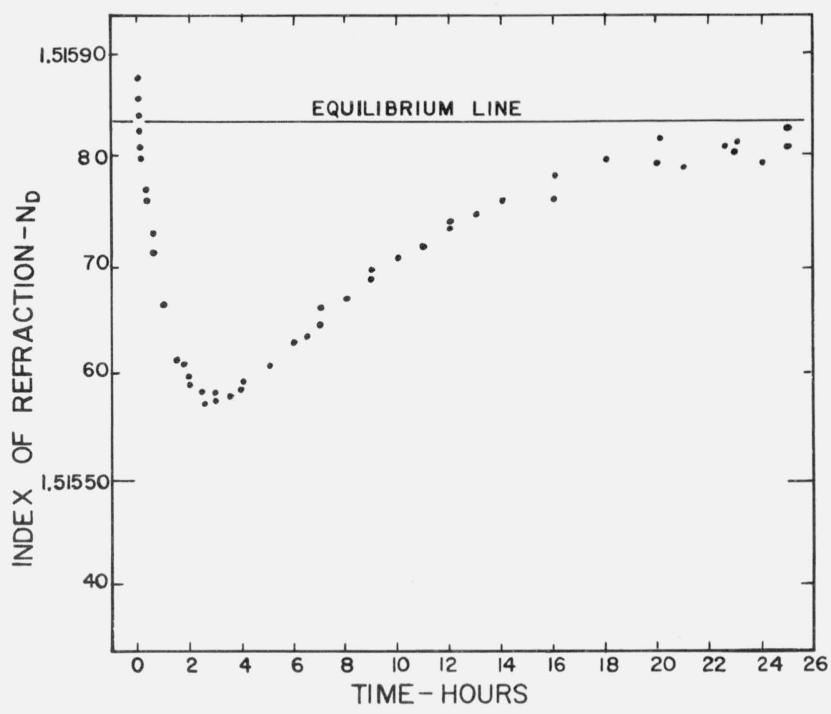

Figure 6. Index versus time for crossover $C$. Equilibrium temperature, $527{ }^{\circ} \mathrm{C}$, approach temperature $477{ }^{\circ} \mathrm{C}$.

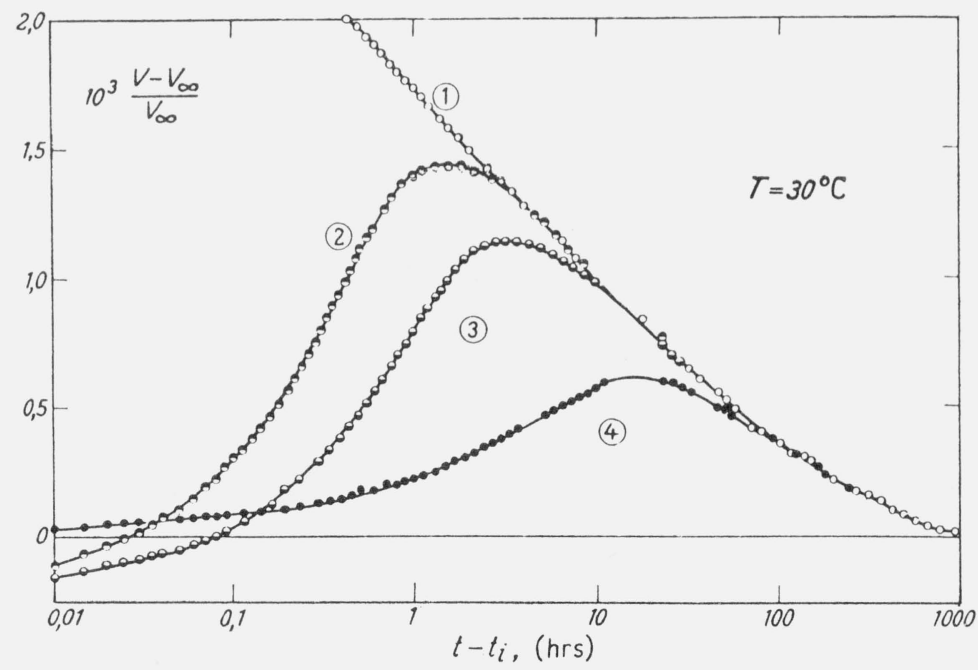

Fig. 24. Evolution isotherme du PVA II après trempe et réchauffement à $30^{\circ} \mathrm{C}$ (Tableau 8)

Tableau 8. Paramètres expérimentaux de la Fig. 24

\begin{tabular}{c|c|c|c|c|c}
\hline $\begin{array}{c}\text { Courbe } \\
\text { no }\end{array}$ & $\begin{array}{c}T_{0} \\
\left({ }^{\circ} \mathrm{C}\right)\end{array}$ & $\begin{array}{c}T_{\mathbf{1}} \\
\left({ }^{\circ} \mathrm{C}\right)\end{array}$ & $\begin{array}{c}t_{\mathbf{1}} \\
(\mathrm{hrs})\end{array}$ & $10^{\mathbf{3} \cdot\left(\boldsymbol{\delta}_{\boldsymbol{i}}-\boldsymbol{\delta}_{\mathbf{1}}\right) \boldsymbol{T}_{\mathbf{1}}}$ & $\begin{array}{c}\boldsymbol{t}^{*} \\
(\mathrm{hrs})\end{array}$ \\
\hline \multirow{2}{*}{1} & 40 & - & - & 0 & - \\
2 & 40 & 10,0 & 160 & 3,2 & 1,4 \\
3 & 40 & 15,0 & 140 & 3,2 & 3,2 \\
4 & 40 & 25,0 & 90 & 3,1 & 18,0
\end{tabular}

FigURE 7. Figure 24 from Kovacs [3]. 
when equilibrium is approached by going from higher to lower indices or densities), the method of this investigation is still applicable. Although in this study the crossover experiment actually involved an intersection between the approach curve and the equilibrium line, all that is actually required is for an approach specimen to be compared with an equilibrium specimen of the same index whether an intersection between the approach curve and equilibrium line takes place or not.

It is interesting to compare the results obtained here with those of Kovacs [3], previously mentioned. The results from his paper are reproduced in figure 7. First, his data involve no crossover. The three curves following the transfer of the specimens from the three different approach temperatures he used, to the equilibrium temperature selected $\left(30{ }^{\circ} \mathrm{C}\right)$ have been superposed by him at "zero" time for comparison. The rise before returning to equilibrium, instead of a dip, as obtained here, comes from the fact that Kovacs was measuring a reciprocal property from the one used here. (Index and density vary concomitantly with annealing $[11,12,13]$.) The sudden rise at the start corresponding to the sudden dip obtained here is not observed because Kovacs plots the logarithm of the time as his abscissa. Kovacs' times before the approach specimen returns to equilibrium are much longer than the ones obtained here. This is to be expected for an organic polymer. Also, the maximum in his rise is a greater proportion of the entire approach curve than the corresponding dip obtained here.

Kovacs concluded from his results that a distribution of relaxation times must be present in the approach curves to equilibrium. If this holds for the material used by him it must also hold for the glass used here; although the shorter times involved before equilibrium is attained by the approach specimens at the crossover indicates that this distribution is considerably narrower for the inorganic glass used here.

\section{References}

[1] H. N. Ritland, Limitations of the fictive temperature concept, J. Am. Ceram. Soc. 39 [12] 403-406 (1956).

[2] S. Spinner and A. Napolitano, Relation between refractive index and elastic moduli of a borosilicate glass after heat treatment, J. Am. Ceram. Soc. 39 [11] 390-94 (1956).

[3] A. J. Kovacs, Glass transition in amorphous polymers. A phenomenological study, Fortschr, Hochpolym-Forsch, 3, 394-507 (1963).

[4] N. M. Brandt, Annealing of 517:645 borosilicate optical glass: I, Refractive index, J. Am. Ceram. Soc. 34 [11]332-38 (1951).

[5] O. W. Grauer, An improved refractometer, NBS Tech. News Bull. 37 (9) 135 (1953); 38 (4) 63 (1954).

[6] Fred A. Molby, Index of refraction and coefficients of expansion of optical glass at low temperature, J. Opt. Soc. Am. 39 [7] 600-11 (1949).

[7] Anuita Winter, Transformation region of glass, J. Am. Ceram. Soc. 26 [6] 189-200 (1943).

[8] H. A. McMaster, Variation of refraction index with time and temperature in annealing region, J. Am. Ceram. Soc. 28 [1] 1-4 (1945).

[9] P. W. Collyer, Study of time and temperature effects on glass in annealing range, J. Am. Ceram. Soc. 30 [11] 338-44 (1947).

[10] (a) B. Daragon, The annealing of flat glass, The Glass Industry 33 [2] 69-74 (1952).

(b) B. Daragon, Annealing curves based on changes in glass structure, Verres et Réfractaires 7 [3] 157-66 (1953).

[11] A. Q. Tool, L. W. Tilton, and J. B. Saunders, Changes caused in refractivity and density of glass by annealing, J. Res. NBS 38 [5] 519-26 (1947); RP1793; Ceram. Abstr., 1951, September, p. 153i.

[12] N. J. Kreidl and R. A. Weidel, Annealing of 517:645 borosilicate optical glass: II, Density, J. Am. Ceram. Soc. 35 [8] 198-203 (1952)

[13] H. N. Ritland, Relation between refractive index and density of a glass at constant temperature, J. Am. Ceram. Soc. 38 [2] 86-88 (1955).

[14] (a) A. Q. Tool, Viscosity and the extraordinary heat effects in glass, J. Res. NBS 37, 73-90 (1946) RP1730; Ceram. Abstr. 1947, January, p. 7.

(b) A. Q. Tool, Relation between inelastic deformability and thermal expansion of glass in its annealing range, J. Am. Ceram. Soc. 29, 240-53 (1946).

(c) A. Q. Tool, Effect of heat-treatment on density and constitution of high-silica glasses of the borosilicate type, ibid 31. 177-86 (1948).

(Paper 70A2-390) 